\title{
LEVANTAMENTO DOS TIPOS DE REPERCUSSÕES RESULTANTES DA AVALIAÇÃO DE DESEMPENHO EM ENFERMAGEM HOSPITALAR ${ }^{1}$
}

\author{
Edméia Assini Balbueno² \\ Márcia Regina Nozawa ${ }^{3}$
}

Balbueno EA, Nozawa MR. Levantamento dos tipos de repercussões resultantes da avaliação de desempenho em enfermagem hospitalar. Rev Latino-am Enfermagem 2004 janeiro-fevereiro; 12(1):58-64.

O presente artigo faz parte de um estudo sobre avaliação de desempenho em enfermagem, e tem como objetivo identificar e descrever as medidas adotadas a partir da avaliação de desempenho do pessoal de enfermagem realizadas em hospitais de municípios da Região do Vale do Paraíba Paulista. Para coleta de dados, aplicou-se questionário aos enfermeiros responsáveis pela direção dos Serviços de Enfermagem ou Serviços de Educação Continuada. Os dados obtidos permitiram verificar que, apesar de 66,7\% dos hospitais não contarem com Serviço de Educação Continuada, 80,9\% realizam avaliação de desempenho e indicaram adotar várias medidas frente aos resultados encontrados. Houve o predomínio da adoção de medidas educativas, tais como: orientação (97\%), treinamento (79,4\%), atualização/reciclagem (70,6\%) e discussão do resultado com o funcionário (88,2\%). Verificou-se, também, que, em uma percentagem significativa dos hospitais, os resultados da avaliação de desempenho oferecem base tanto para as promoções (20,6\%), oferta de diversos incentivos (58,8\%), quanto para as demissões de funcionários (58,8\%).

DESCRITORES: administração em enfermagem; recursos humanos em saúde; avaliação de desempenho

\section{SURVEY OF TYPES OF REPERCUSSIONS AS A RESULT OF} PERFORMANCE EVALUATION IN HOSPITAL NURSING

This article is part of a study about performance evaluation in nursing and aims to identify and describe the measures adopted on the basis of the nursing staff performance evaluation that was carried out at municipal district hospitals in the Paraiba Valley Region - São Paulo, Brazil. Data were collected by means of a questionnaire, which was applied among the nurses in charge of the Nursing or Continuous Education Services. The obtained data allowed us to verify that, although $66.7 \%$ of the hospitals do not have a Continuous Education Service, $80.9 \%$ carry out performance evaluation and indicate the adoption of several measures according to the observed results. Educational measures prevailed, such as: orientation (97.0\%), training (79.4\%), updating/recycling (70.6\%) and discussion of the result with the employee (88.2\%). It was also verified that, in a significant percentage of the hospitals, the performance evaluation results do not only offer support for promotions (20.6\%) and several incentives (58.8\%), but also for employees' dismissal (58.8\%).

DESCRIPTORS: nursing administration; human resources in health manpower; performance evaluation

\section{LEVANTAMIENTO DE LOS TIPOS DE REPERCUSIONES RESULTANTES DE} LA EVALUACIÓN DE DESEMPEÑO EN ENFERMERIA HOSPITALARIA

El presente artículo hace parte de un estudio sobre evaluación de desempeño en enfermería; tiene como objetivo identificar y describir las medidas adoptadas a partir de la evaluación de desempeño del personal de enfermería realizadas en hospitales de municipios de la Región del "Vale do Paraíba Paulista". Para recolectar los datos se aplicó un cuestionario a los enfermeros responsables por la dirección de los Servicios de Enfermería o Servicios de Educación Continuada. Los datos obtenidos permitieron verificar que a pesar de $66,7 \%$ de los hospitales no contar con un Servicio de Educación Continuada, $80,9 \%$ realizan evaluación de desempeño e indicaron adoptar varias medidas frente a los resultados encontrados. Hubo el predominio en la adopción de medidas educativas, tales como: orientación (97,0\%), entrenamiento (79,4\%), actualización $(70,6 \%)$ y discusión del resultado con el funcionario (88,2\%). Se notó, también que en un porcentaje significativo de los hospitales, los resultados de la evaluación de desempeño ofrecen la base tanto para las promociones (20,6\%), oferta de diversos incentivos (58,8\%), como para las demisiones de funcionarios (58,8\%).

DESCRIPTORES: administración en enfermería; recursos humanos en salud; evaluación del rendimiento de empleados

\footnotetext{
${ }^{1}$ Este artigo é parte da dissertação de mestrado intitulada: "Análise da avaliação de desempenho em enfermagem hospitalar na Região do Vale do Paraíba Paulista" apresentada à Pós-Graduação da Faculdade de Ciências Médicas da Universidade Estadual de Campinas, 2001; ${ }^{2}$ Professor Assistente II da Universidade de Taubaté, e-mail: balbueno@bol.com.br; ${ }^{3}$ Professor Assistente Doutor da Faculdade de Ciências Médicas da Universidade Estadual de Campinas
} 
Oexercício continuado de supervisão de alunos de graduação em enfermagem, durante os estágios de administração em enfermagem hospitalar, tem permitido identificar que os enfermeiros apresentam dificuldades na elaboração de estratégias de avaliação de desempenho e que as medidas adotadas, frente aos resultados das avaliações, eventualmente realizadas, são muitas vezes incoerentes com as diferentes situações encontradas. Alguns funcionários são transferidos ou remanejados, mas, muitas vezes, permanecem realizando as mesmas atividades, mesmo que seu desempenho tenha sido considerado insatisfatório. Essa constatação leva a questionamentos acerca da valorização efetiva e dos usos concretos da avaliação de desempenho em enfermagem, tendo em vista que a defesa da qualidade da assistência tem sido discurso freqüentemente enunciado pelos profissionais, entidades de classe e órgãos formadores em enfermagem.

Autores argumentam que: "A avaliação de desempenho, amplamente empregada em muitas empresas, tem se tornado motivo de preocupação, visto que sua utilização acontece seguindo critérios e modelos provenientes de outras instituições, nem sempre respeitando, no entanto, os aspectos peculiares de cada organização, no que se refere à cultura, valores, filosofia e política de pessoal”(1).

Essa situação tem gerado inúmeras discussões e polêmicas, entre professores de enfermagem, profissionais da área e administradores em geral, em torno da qualidade do trabalho desenvolvido pelo pessoal de enfermagem, qualidade essa relacionada diretamente ao processo de desenvolvimento dos recursos humanos ${ }^{(2)}$.

Clientes e usuários estão cada vez mais exigentes com os produtos e serviços de que dispõem, e a questão da qualidade tem apresentado crescente preocupação em todo o mundo. A nova consciência de qualidade dos produtos e serviços concede mais valorização aos esforços do indivíduo, considerando-se que as pessoas envolvidas são fundamentais, pois a qualidade depende do trabalho individual ou em grupo ${ }^{(3)}$.

A qualidade tornou-se, na atualidade, palavra de sobrevivência, levando as instituições de saúde a repensarem suas estruturas, processos e relações de trabalho. Cresce, a cada dia, a procura por profissionais capacitados para a detecção de falhas e proposição de soluções aos problemas encontrados, e também preparados para o trabalho em equipe, numa perspectiva participativa, cooperativa e sinérgica ${ }^{(4)}$.

O posicionamento adotado pelas organizações na oferta de produtos e serviços de qualidade, como também a consciência da importância dos recursos humanos na obtenção de qualidade, além da busca por redução de custos, caracteriza atitude coerente que Ihes permitirá condições e possibilidades de sobrevivência no mercado atual.

A preocupação dos hospitais, atualmente, pela busca da qualidade dos serviços vem exigindo de seus profissionais e colaboradores uma nova postura. Dessa forma, a importância da procura pela capacitação profissional e aprimoramento das habilidades dos funcionários é condição para garantia da sobrevivência e manutenção das organizações ${ }^{(5)}$.

$\mathrm{Na}$ prática da enfermagem, a qualidade da assistência prestada ao usuário decorre diretamente do desempenho de quem a executa ${ }^{(6)}$.

Promover ações que associem o desenvolvimento profissional dos integrantes da equipe aos objetivos da organização, utilizando métodos que direcionem o desempenho para alcance de melhores resultados, tornase cada vez mais necessário ${ }^{(5)}$.

Considerando-se a importância da relação entre a qualidade dos serviços de saúde e o desempenho do pessoal de enfermagem, a avaliação desse desempenho deveria ser uma preocupação constante das organizações hospitalares na direção da promoção do desenvolvimento dos recursos humanos para garantir a qualidade da assistência oferecida.

A avaliação de desempenho na enfermagem é considerada como meio para: "melhorar a qualidade dos cuidados de Enfermagem prestados; melhorar a organização e o funcionamento dos serviços de Enfermagem assim como desenvolver as capacidades e potencialidades do enfermeiro; e contribuir para valorização pessoal e profissional do enfermeiro"(7).

Segundo alguns autores, pela avaliação de desempenho ou a partir de seus resultados, as organizações decidem o futuro e destino de seus recursos humanos: pessoas são admitidas, promovidas, dispensadas, remanejadas, treinadas ou advertidas. Portanto, sua utilização deve ser feita com a máxima seriedade, competência e mediante uso de critérios racionais e objetivos de julgamento. Caso contrário, as 
decisões tomadas sob influência da subjetividade podem afetar injustamente os avaliados, além de, muitas vezes, implicarem custos adicionais e desnecessários à empresa $^{(8-9)}$.

Diante do exposto, é necessário, na área de enfermagem, a utilização de estratégias específicas para a melhoria da qualidade de seus serviços, como um processo que promova crescimento e desenvolvimento pessoal e profissional ${ }^{(2)}$.

Autores salientam que, para garantir o desenvolvimento do pessoal, o serviço de enfermagem deve contar com um setor que agrupe, organize e coordene as atividades educacionais, sistematizando e articulando todos os setores da enfermagem na formação de programas de desenvolvimento de pessoal. Para que a avaliação de desempenho não seja conduzida como atividade isolada, deve-se garantir a interação de todos os envolvidos no processo. Essa tarefa é freqüentemente assumida ou coordenada por setores denominados: serviço de educação contínua ou continuada, educação em serviço, ou setor de treinamento ${ }^{(10)}$.

Tomando o processo de avaliação de desempenho como parte integrante do processo ensino-aprendizagem e considerando-se a prática de enfermagem como processo educativo, sua utilização de forma contínua e sistematizada poderá contribuir para o alcance ou melhoria da qualidade da assistência.

A avaliação de desempenho objetiva: adequar o indivíduo ao cargo, orientar treinamentos de pessoal, decidir promoções, melhorar as relações de trabalho, autoaperfeiçoamento, coletar informações básicas para pesquisa de recursos humanos, identificar estimativas do potencial do indivíduo, estimular aumento de produtividade, conhecer o desempenho organizacional, retroalimentar o avaliado, decidir transferências e demissões de funcionários ${ }^{(11)}$.

Para que os resultados da avaliação de desempenho tragam benefícios a todos que participam do processo, seja o avaliado, o avaliador ou a organização, devem permitir identificar as habilidades específicas que necessitam ser desenvolvidas ou adquiridas pelo avaliado e, também, promover a melhoria da qualidade de vida no trabalho. Dessa forma, torna-se necessário transformar os sistemas de avaliação utilizados em instrumentos úteis a todos seus usuários, como ferramentas de prevenção, identificação e resolução de problemas ${ }^{(12)}$.

A utilização da avaliação de desempenho está relacionada diretamente ao processo de desenvolvimento de pessoal. Sua importância é comprovada na mensuração e retroalimentação, no recrutamento e seleção, no treinamento e aperfeiçoamento, auxiliando nas justificativas de promoção, punição, transferências e desligamentos, como também na melhoria do relacionamento interpessoal ${ }^{(13)}$.

$\mathrm{Na}$ área de enfermagem, em estudo realizado em hospitais gerais do município de São Paulo, no ano de 1983, verificou-se que a maioria deles utilizava os resultados das avaliações como justificativas para a admissão, demissão e promoção do pessoal, embora as enfermeiras pesquisadas considerassem que os resultados deveriam ser utilizados para programas de desenvolvimento de pessoal ${ }^{(14)}$.

Acredita-se que o conhecimento das medidas adotadas nos hospitais de municípios da Região do Vale do Paraíba Paulista, frente aos resultados das avaliações realizadas, é de suma importância, pois possibilitará a identificação de subsídios para o preparo e formação de novos profissionais. Além disso, pode estimular reflexão e possíveis intervenções dos enfermeiros e administradores de recursos humanos nos hospitais estudados, bem como de outros profissionais envolvidos com essa temática.

Este estudo teve por objetivo identificar e descrever as medidas adotadas a partir da avaliação de desempenho do pessoal de enfermagem realizadas em hospitais de municípios da Região do Vale do Paraíba Paulista.

\section{MÉTODO}

Trata-se de pesquisa descritiva com a adoção de método de raciocínio indutivo.

O campo de estudo compreendeu 39 municípios da Região do Vale do Paraíba Paulista. Pelo Decreto $\mathrm{n}^{\circ}$ 40397/1995, a essa região pertencem as Diretorias Regionais de Saúde (DIR) XXI de São José dos Campos, com 12 municípios e a DIR XXIV de Taubaté, com 27 municípios $^{(15)}$.

A população informante foi composta pelos enfermeiros responsáveis pela Direção dos Serviços de Enfermagem ou pelos Serviços de Educação Continuada dos hospitais localizados nos municípios das DIR XXI e DIR XXIV, e totalizou um conjunto de 42 profissionais.

Utilizou-se a técnica de observação direta extensiva, optando-se pelo questionário como instrumento 
de coleta de dados, por tratar-se de instrumento que permite a participação direta dos sujeitos da pesquisa, sem a presença do entrevistador.

O questionário compôs-se de 11 perguntas, dentre as quais, três fechadas, cinco abertas e três mistas, ou seja, combinando o formato de múltipla escolha e campo aberto para manifestação de opiniões. Abrangia questões relativas às características da população e da instituição; serviço de educação continuada; prática da avaliação de desempenho; medidas adotadas para promover, punir e dispensar o funcionário e tipos de incentivos e punições adotados.

A coleta de dados, conduzida pessoalmente por uma das pesquisadoras, ocorreu no período de janeiro a fevereiro de 2001.

O projeto da pesquisa foi apresentado ao Comitê de Ética em Pesquisa da Universidade de Taubaté, tendo sido aprovado em 10 de março de 2000. A coleta de dados seguiu as normas da Resolução 196/96 do Conselho Nacional de Saúde, relativa à pesquisa com seres humanos (16). A autorização dos administradores dos hospitais selecionados e a anuência dos sujeitos pesquisados foram obtidas, respectivamente, mediante Termo de Consentimento da Instituição e Termo de Consentimento Livre e Esclarecido de cada enfermeiro que participou do estudo.

\section{RESULTADOS E DISCUSSÃO}

\section{Caracterização da população e dos hospitais}

Em relação à população estudada, verificou-se que a maioria $40(95,2 \%)$ é composta por mulheres, distribuídas principalmente nas seguintes faixas etárias: 25 (59,6\%) entre 26 e 37 anos, 14 (33,3\%) acima de 37 anos, sendo que apenas um (2,4\%) encontra-se abaixo de 25 anos e dois (4,7\%), não informaram a idade.

A maioria dos enfermeiros, 33 (78,6\%), responde pela direção dos serviços de enfermagem, apenas três (7,1\%) são encarregados do Serviço de Educação Continuada, e os outros seis (14,3\%) ocupam o cargo de Supervisor, Vice-Diretor ou Diretor Administrativo e respondem pelo Serviço de Enfermagem.

Evidenciou-se que 20 (47,6\%) enfermeiros graduaram-se na Universidade de Taubaté, e dez (23,8\%), na Escola de Enfermagem Wenceslau Braz. Outros dez
(23,6\%) concluíram graduação em diferentes escolas, e dois $(4,8 \%)$ não ofereceram essa informação. A maioria dos enfermeiros é originária de escolas da capital ou dos municípios do interior do Estado de São Paulo; somente duas escolas situam-se em outros Estados da Federação. Entre elas, destaca-se a participação expressiva de uma escola localizada em Itajubá, Minas Gerais, situada a 137 km da Região do Vale do Paraíba Paulista, na qual dez $(23,8 \%)$ enfermeiros cursaram a graduação em enfermagem.

Os dados obtidos reforçam a importância da análise da prática da avaliação de desempenho em enfermagem nos hospitais da Região do Vale do Paraíba Paulista, por ser um mercado de trabalho que absorve grande parte dos profissionais graduados pela universidade na qual uma das autoras atua profissionalmente como docente.

A maior parte dos enfermeiros está formada há mais de cinco anos, sendo a maior ocorrência 12 (28,6\%) entre seis e dez anos, seguida dos profissionais com 16 a 20 anos, sete $(16,6 \%)$, seis (14,3\%) entre 11 e 15 anos, apenas seis $(14,3 \%)$ possuem até cinco anos de formados, seis $(14,3 \%)$ nada informaram e cinco $(11,9 \%)$ estão formados há mais de 21 anos. Esse fato permite considerar que não se trata de profissionais recémgraduados, mas que acumulam tempo de experiência profissional relativamente expressivo.

No que diz respeito ao aperfeiçoamento profissional, após a graduação, a maior parte dos enfermeiros $28(66,7 \%)$ concluiu algum curso de especialização; apenas dois $(4,8 \%)$ cursaram pósgraduação stricto sensu; um concluiu o mestrado, e outro, doutorado.

Utilizando a classificação do porte hospitalar a partir do número de leitos oferecidos ${ }^{(17)}$, ou seja, pequeno porte (até 50 leitos), de médio porte (51 a 150 leitos), de grande porte (151 a 500 leitos) e de porte especial (acima de 500 leitos), observou-se que 19 (45,2\%) hospitais são de médio porte; dez $(23,8 \%)$, de pequeno porte; seis $(14,3 \%)$, de grande porte; e sete $(16,7 \%)$ não foram classificados, por ausência de informação.

Quanto ao tipo de organização hospitalar, verificouse que 19 (45,2\%) são hospitais privados; 16 (38,1\%) são hospitais filantrópicos ou privados sem fins lucrativos; e dos sete $(16,6 \%)$ restantes, três $(7,1 \%)$ são públicos (estadual e municipal), dois (4,8\%) são fundações, e dois $(4,8 \%)$ nada informaram. Desse conjunto total, 28 (66,7\%) 
são hospitais conveniados ao Sistema Único de Saúde (SUS), e os demais, 14 (33,3\%), correspondem a hospitais exclusivamente privados.

Procurou-se identificar o Serviço de Educação Continuada nos hospitais estudados, tendo em vista que esse serviço é o responsável pela coordenação de programas educacionais para promover desenvolvimento dos recursos humanos e que desempenha as mais variadas atividades.

Verificou-se que $28(66,7 \%)$ dos hospitais não possuem esse serviço e que $13(30,9 \%)$ possuem. Apenas um $(2,4 \%)$ não informou a existência desse serviço.

Constatou-se que, apesar de a maior parte dos hospitais não contar com Serviço de Educação Continuada, os enfermeiros indicaram desenvolver várias atividades educacionais, denotando um certo interesse, preocupação ou iniciativa desses profissionais com 0 desenvolvimento do pessoal de enfermagem, mesmo na ausência de um serviço estruturado para tal fim.

Medidas adotadas frente aos resultados das avaliações realizadas

A utilização dos resultados da avaliação é considerada parte fundamental no processo avaliativo. Esses servem de subsídios para decisões administrativas relativas ao destino dos recursos humanos dentro das organizações.

As medidas adotadas frente aos resultados das avaliações devem ser coerentes, para evitar a subitilização dos mesmos e a ocorrência de erros, injustiças e distorções nas decisões, o que pode interferir na qualidade dos serviços prestados.

Em estudo ${ }^{(14)}$ sobre avaliação de desempenho de enfermeiras nos hospitais gerais do município de São Paulo, concluiu-se que a participação efetiva das enfermeiras nos programas de avaliação é feita principalmente por meio de discussão após a avaliação.

Da totalidade dos enfermeiros estudados, 34 $(80,9 \%)$ deles indicaram desenvolver algum tipo de avaliação, e apontaram as medidas adotadas frente aos resultados obtidos.

Ressalta-se que os enfermeiros dos hospitais estudados indicaram a adoção de mais de uma medida posterior à avaliação de desempenho do pessoal de enfermagem. Dentre as medidas adotadas, destaca-se a orientação, em 33 (97\%) indicações; a discussão com o interessado, em $30(88,2 \%)$ indicações; o treinamento, em 27 (79,4\%); e o desenvolvimento de programas de atualização/reciclagem, em 24 (70,6\%) citações. Os resultados das avaliações são utilizados como justificativas para a demissão por 20 (58,8\%) dos enfermeiros; para a concessão de incentivos diversos, por 20 (58,8\%); e para a promoção de funcionários, por sete $(20,6 \%)$ enfermeiros.

Os dados relativos aos aspectos citados pelos enfermeiros de hospitais da região, utilizados para a promoção, punição e dispensa de funcionários, tipos de incentivos e punições adotadas, envolvem os 42 hospitais que participaram do estudo, visto que mesmo aqueles que não realizam avaliação de desempenho citaram aspectos utilizados para tal fim.

Para efeito de análise, os aspectos citados pelos enfermeiros, para promoção, punição ou dispensa de funcionários, foram agrupados nas áreas de Conhecimento, Habilidade e Atitude, em consideração à importância desses conjuntos de indicadores na avaliação e desenvolvimento do potencial humano.

Verificou-se que os enfermeiros utilizam com maior freqüência os aspectos relativos a: comportamento/ atitudes dos funcionários (51,1\% para promover; $70 \%$ para punir e $56,2 \%$ para dispensar), habilidades (24,5\% para promover; $13,3 \%$ para punir e $16,8 \%$ para dispensar), a outros tipos de critérios/aspectos (13,8\% para promover; $16,7 \%$ para punir e $23,6 \%$ para dispensar) e ao conhecimento (10,6\% para promover; $0 \%$ para punir e $3,4 \%$ para dispensar).

As respostas dos enfermeiros relativas aos tipos de incentivos adotados pelos hospitais estudados foram agrupadas em três categorias específicas: incentivos Profissionais, Psicossociais e Monetários, considerandose as respostas dadas, para permitir análise e discussão dos resultados.

Evidenciou-se maior freqüência na utilização de incentivos do tipo profissional, 78,6\%, tais como: "cursos, palestras, promoção de cargo, reciclagem". Os incentivos psicossociais elogios, folgas extras, carta de agradecimento, confraternizações foram citados em 59,5\% das respostas, e os monetários, em $40,5 \%$ das respostas. Nesses, incluíram-se os seguintes elementos de incentivo: "bolsa de estudos, pagamento de cursos, convênios, vale transporte", entre outros.

A utilização de incentivos profissionais parece vincular-se a uma preocupação com desenvolvimento 
cognitivo dos membros da equipe de enfermagem. Já a utilização de incentivos psicossociais e monetários, por praticamente metade deles $(59,5 \%$ e $40,5 \%$, respectivamente), pode estar associada à preocupação com o bem-estar e melhoria da auto-estima do funcionário na instituição.

Entre os tipos de punições adotadas, verificou-se que os hospitais obedecem a uma certa sistemática para punir seus funcionários: 10 (23,8\%) hospitais adotam a advertência verbal, escrita e suspensão; sete (16,7\%) indicam adotar a advertência verbal, escrita, suspensão e demissão; seis $(14,3 \%)$ adotam a advertência e suspensão; três $(7,1 \%)$ utilizam a advertência verbal e escrita; dois (4,8\%), advertência verbal, escrita e demissão; dois $(4,8 \%)$ de acordo com a Consolidação das Leis Trabalhistas; dois $(4,8 \%)$ e advertência, suspensão e dispensa e três $(7,1 \%)$ não informaram.

Outros tipos de punições foram também citados pelos enfermeiros de sete hospitais (16,7\%), embora menos freqüentemente: "advertência escrita e suspensão"; "advertência com redução de benefícios e suspensão"; "advertência verbal, suspensão e redução do período de férias"; "advertência verbal, escrita e educativa"; "advertência escrita"; "perda da cesta básica após falta injustificada"; e "advertências verbais, escritas, suspensão e troca de plantão".

\section{CONCLUSÕES E CONSIDERAÇÕES FINAIS}

Os dados obtidos, com base nos relatos sobre as medidas adotadas por enfermeiros dos hospitais de municípios da região do Vale do Paraíba Paulista, frente aos resultados das avaliações realizadas, permitiram verificar que:

- apesar de a maioria dos hospitais não contar com Serviço

\section{REFERÊNCIAS BIBLIOGRÁFICAS}

1. Gonçalves VLM, Leite MMJ. Sentimentos dos enfermeiros no processo de avaliação de desempenho da equipe de enfermagem de um hospital universitário. O Mundo da Saúde 1999; 23 (3):167-73.

2. Balbueno EA. Análise da avaliação de desempenho em enfermagem hospitalar na Região do Vale do Paraíba Paulista. [Dissertação]. Campinas (SP): Faculdade de Ciências Médicas/UNICAMP; 2001.

3. Moller C. O lado humano da qualidade: maximizando a qualidade dos produtos e serviços através do desenvolvimento das pessoas. 12. ed. São Paulo (SP): Pioneira; 1999. de Educação Continuada, a maior parte deles realiza avaliação de desempenho e indicou adotar várias medidas frente aos resultados encontrados;

- entre as medidas adotadas, predominou a adoção de medidas educativas, tais como: orientação, treinamento, atualização/reciclagem e discussão com o funcionário;

- em uma percentagem significativa dos hospitais, os resultados das avaliações oferecem base tanto para promoções e oferta de diversos incentivos quanto para as demissões de funcionários;

- para justificar as promoções, punições e demissões, apóiam-se, com maior freqüência, em aspectos relativos ao comportamento e atitudes dos funcionários;

- os incentivos mais utilizados para motivação dos funcionários são profissionais, seguidos pelos psicossociais e monetários.

Este estudo permitiu o reconhecimento de que as conseqüências da avaliação de desempenho em enfermagem, nas instituições hospitalares estudadas, materializam-se em medidas de controle que incidem sobre o trabalhador, em detrimento da análise dos resultados de um trabalho que sempre é realizado coletivamente. Possibilitou, também, reflexões sobre a necessidade de revisão crítica de práticas nesse âmbito.

Assim, o enfermeiro que gerencia a assistência de enfermagem deve estar preparado para implementar a avaliação de desempenho como um processo que visa o aprimoramento e desenvolvimento de recursos humanos ${ }^{(2)}$.

É imprescindível, portanto, que esta temática esteja presente no conteúdo dos cursos de graduação, em particular nas disciplinas de Administração em Enfermagem, objetivando preparar os futuros profissionais na implementação de programas de avaliação de desempenho coerentes com a busca da qualidade da assistência dos serviços prestados e com o desenvolvimento do profissional de enfermagem ${ }^{(2)}$.

4. Silva MJP. Valor da comunicação para o sucesso dos processos de qualidade. In: Mello JB, Camargo MD. Qualidade na saúde. Práticas e Conceitos. Normas ISO nas Áreas Médico-Hospitalar e laboratorial. São Paulo (SP): Best Seller; 1998. p. 69-76.

5. Ferreira CC, Marra CC. Avaliação de desempenho humano: importância na enfermagem. Rev Paul Enfermagem 2001; 20(2):25-32.

6. Ciosak SI. Avaliação de desempenho e o aprimoramento de enfermeiros: expectativas do avaliador e avaliado. [Tese]. São Paulo (SP): Escola de Enfermagem/USP; 1994.

7. Soares A, Lopes A, Nunes L, Gonçalves L. Reflectindo sobre o relatório crítico de actividades. Rev Tec Enfermagem Nurs 1997; 10(117):35-8. 
8. Aquino CP. Avaliação de desempenho: formulação básica. Rev Paul Hosp 1979; 27(9):284-90.

9. Lucena MDS. Avaliação de desempenho. São Paulo (SP): Atlas; 1992.

10. Leite MMJ, Pereira LL. Educação continuada em enfermagem. In: Kurcgant P, Cunha KC, Massarollo MCKB, Ciampone MHT, Silva VEFS, Castilho V, et al., coordenadoras. Administração em enfermagem. São Paulo: EPU; 1991. p. 147-3.

11. Chiavenato I. Avaliação de desempenho. 2. ed. comp. São Paulo (SP): Atlas; 2000.

12. Castro GAO, Lima GBC, Veiga MRM. Implantação de um sistema de avaliação de desempenho: métodos e estratégias. Rev Admin 1996; 31(3):38-52.

13. Bezerra ALQ, Silva MB. Avaliação de desempenho em enfermagem. Rev Paul Enfermagem 1993; 12(3):62-4.

14. Kurcgant P. Estudo sobre avaliações de desempenho de enfermagem nos hospitais gerais do município de São Paulo, 1983. [Tese]. São Paulo (SP): Escola de Enfermagem/USP; 1984.

15. Decreto n. 40397 de 23 de outubro de 1995. Dispõe sobre alterações nas Diretorias Regionais de Saúde que especifica, organiza os Núcleos Regionais de Saúde e dá providencias correlatas. Diário Oficial do Estado de São Paulo 1995; seção I, p. 1-2.

16. Resolução n. 196, de 10 de outubro de 1996. Diretrizes e normas regulamentadoras de pesquisas envolvendo seres humanos. Bioética 1996; 4(2 supl):15-25.

17. Ministério da Saúde (BR). Secretaria de Estado da Saúde. Terminologia Básica para atividades de auditoria e controle. São Paulo: SUS; 1998. 